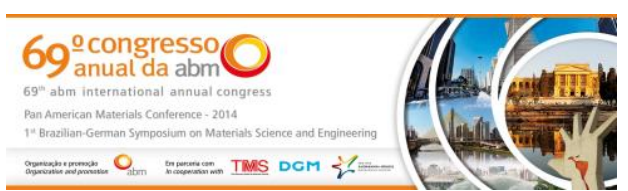

Tema: Diagramas de fases e transformações de fases

\title{
ESTUDO EXPERIMENTAL DO AVANÇO DE INCLUSÕES NÃO-METÁLICAS ATRAVÉS DO TRATAMENTO COM CÁLCIO*
}

\section{Resumo}

\author{
Bruna lost Camacho \\ Julio Aníbal Morales Pereira² \\ Wagner Viana Bielefeldt ${ }^{3}$ \\ Antônio Cezar Faria Vilela ${ }^{4}$
}

Inclusões não metálicas são impurezas presentes nos aços que corrompem, quando não moderadas, suas propriedades mecânicas e metalúrgicas. O tratamento de inclusões com ligas à base de cálcio é fortemente utilizado visando à produção de aços mais limpos e é fortemente induzido pela composição química e temperatura do aço. O objetivo geral deste trabalho será o estudo termodinâmico e experimental a respeito da modificação de inclusões não metálicas através do tratamento com cálcio, em aços de diferentes teores de enxofre. $O$ presente trabalho utilizará como metodologia: i) obtenção de dados através de experimentos em forno resistivo para determinar os dados iniciais utilizados nas simulações; ii) estudo termodinâmico computacional através do software FactSage; iii) análise de inclusões via MEV/EDS e ASPEX-PSEM explorer. iv) comparação dos resultados obtidos nos dois meios de análise e também compará-las com os resultados obtidos via simulação. Para tanto, serão estabelecidas dife rentes temperaturas e concentrações de cálcio no banho e diferentes tempos de processo. Como resultado final, espera-se gerar um maior conhecimento que contribua para a compreensão dos fenômenos envolvidos em aços com maior teor de enxofre. Além disso, espera-se validar as técnicas e metodologia empregadas neste trabalho.

Palavras-chave: Inclusões não-metálicas; Cálcio; FactSage.

\section{EXPERIMENTAL STUDIES OF NON - METALLIC INCLUSIONS THROUGH THE TREATMENT WITH CALCIUM}

\section{Abstract}

Non-metallic inclusions are impurities present in steels and can affect their mechanical and metallurgical properties whether not controlled. The treatment of inclusions with calcium-based alloys is strongly used to produce cleaner steels. This treatment is strongly influenced by the chemical composition and temperature of the steel. The general goal of this work is to perform the thermodynamic and experimental study about the modification of non-metallic inclusions by treatment with calcium in steels with different sulfur contents. This study will use as methodology: i) obtaining data through experiments in lab resistive furnace to determine the initial data used in the simulations, ii) to perform a computational thermodynamic study through the software FactSage iii) inclusions analysis via SEM / EDS and ASPEX-PSEM explorer, iv) comparison and discussion of the results obtained in both analyses in order to compare them with the results obtained through the simulation. For this, will be considered different temperatures and concentrations of calcium in the bath and different processing times. The final result is expected to generate a greater knowledge that contributes for the comprehension about the phenomena involved in steels with higher sulfur content. It is hoped to validate the techniques and methodology used on this paper.

Keywords: Non-metallic inclusions; Calcium; FactSage.

1 Graduanda em Engenharia Metalúrgica, Laboratório de Siderurgia, UFRGS, Porto Alegre, RS, Brasil; bicamacho@gmail.com.

2 Eng, Dr., Laboratório de Siderurgia, LASID, UFRGS, Porto Alegre, RS, Brasil.

3 Eng, Dr., Laboratório de Siderurgia, LASID, Depto. de Metalurgia, UFRGS, Porto Alegre, RS, Brasil, wagner@ct.ufrgs.br.

4 Eng, Dr., Laboratório de Siderurgia, LASID, Depto. de Metalurgia, UFRGS, Porto Alegre, RS, Brasil.

\footnotetext{
* Contribuição técnica ao 69ำ Congresso Anual da ABM - Internacional e ao 14ํㅡㄹ ENEMET - Encontro Nacional de Estudantes de Engenharia Metalúrgica, de Materiais e de Minas, 21 a 25 de julho de 2014, São Paulo, SP, Brasil.
} 


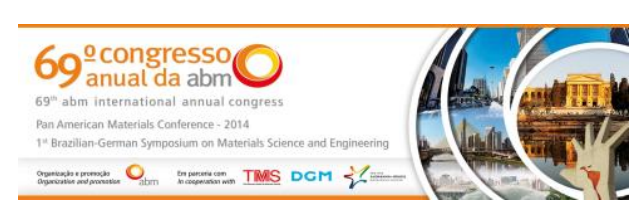

\section{INTRODUÇÃO}

Aços com teor elevado de enxofre possuem alta usinabilidade e tem sido amplamente aplicados na produção de peças para a indústria automobilística. Todavia, seu teor final de oxigênio não é satisfatório para uso em componentes de alto desempenho.

Ao escolher o elemento desoxidante deve-se relacionar o teor de oxigênio residual com o grau de limpeza do aço desejado. Dessa forma, o alumínio é uma opção largamente utilizada para reduzir o teor de oxigênio desse material, mesmo que seu uso como desoxidante resulte na formação de inclusões não-metálicas sólidas que, por sua vez, podem causar obstrução de válvulas no lingotamento contínuo.

Conforme Holappa et al. [1], o tratamento com cálcio é um método bem estabelecido para transformar óxidos e sulfetos no aço em inclusões menos prejudiciais que podem gerar efeitos benéficos nas propriedades. A transformação de clusters sólidos de $\mathrm{Al}_{2} \mathrm{O}_{3}$ em cálcio-aluminatos líquidos através do tratamento com cálcio é uma técnica largamente empregada afim depara reduzir a obstrução em válvulas de lingotamento continuo na produção de aços acalmados ao alumínio. O tratamento com cálcio é ainda um método chave para melhora da usinabilidade, que também pode ser melhorada através do aumento do teor de enxofre.

A forma dos cavacos é determinante na velocidade de usinagem. Metais, ao serem usinados, devem produzir um cavaco frágil e, portanto, quebradiço, o que propicia uma maior facilidade de manuseio e operação [2]. A adição de enxofre ao aço é muito conhecida e utilizada a fim de conduzir a formação de sulfetos de manganês. Tais inclusões possuem baixo ponto de fusão e alta deformabilidade. $O$ efeito é positivo na usinabilidade, ao agirem como lubrificantes no corte, facilitando a quebra do cavaco na zona de cisalhamento. Assim como no emprego do alumínio no refino, o emprego do enxofre como formador de sulfetos é agente causador de inclusões não-metálicas. As características termodinâmicas e cinéticas do tratamento de inclusões nãometálicas com cálcio são fortemente influenciadas pela composição química do aço e temperatura, porém ainda não são completamente conhecidas. Em estudo a respeito da modificação dos óxidos pelo cálcio, Larsen e Fruehan [3] compararam dados experimentais com a prática industrial e relataram que a predição teórica baseada em dados termodinâmicos concorda com os resultados das amostras de corridas executadas em laboratório e na indústria. Indicando que as reações entre o cálcio dissolvido e as inclusões são relativamente rápidas. Ainda no mesmo trabalho fazem duas importantes considerações: que para se obter a completa modificação dos óxidos para cálcio-aluminatos líquidos em aços acalmados ao alumínio, o teor de enxofre deve ser muito baixo e que quanto maior a temperatura de lingotamento mais fácil é a formação de inclusões líquidas.

O presente trabalho aborda um estudo termodinâmico e experimental a respeito da modificação de inclusões não metálicas através do tratamento com cálcio nos aços SAE 1141 e SAE 1117, objetivando especificamente: 1) obter o perfil inclusionário do aço após o tratamento em questão; 2) realizar a comparação de dados obtidos via simulação termodinâmica com dados experimentais e; 3) discutir diferentes configurações de temperaturas e composição química do aço para esse modelo de estudo.

\section{MATERIAIS E MÉTODOS}

\footnotetext{
* Contribuição técnica ao $69^{\circ}$ Congresso Anual da ABM - Internacional e ao 14ํㅡㄹ ENEMET - Encontro Nacional de Estudantes de Engenharia Metalúrgica, de Materiais e de Minas, 21 a 25 de julho de 2014, São Paulo, SP, Brasil.
} 


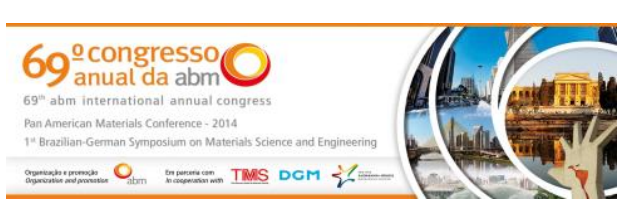

Os aços estudados são o SAE 1141 e o SAE 1117. Classificados pela SAE-AISI como aços carbono ressulfurados, tem sido amplamente aplicados na produção de peças para a indústria automobilística. São conhecidos por sua boa usinabilidade, temperabilidade, conformabilidade e baixa soldabilidade. Nas tabelas 1 e 2 verifica-se a faixa de concentração dos principais elementos de liga dos aços em questão.

A metodologia é baseada em estudo realizado anteriormente por Bielefeldt [4], e a respeito do aço SAE 1117 os autores se referem ao trabalho Kirichenco [5].

Tabela 1. Composição química para o aço SAE 1141 em \% em massa.

\begin{tabular}{|c|c|c|c|}
\hline $\mathrm{C}$ & $\mathrm{Mn}$ & $\mathrm{S}$ & $\mathrm{P}$ \\
\hline $0,37-0,45$ & $1,35-1,65$ & $0,08-0,13$ & 0,040 máx. \\
\hline
\end{tabular}

Tabela 2. Composição química para o aço SAE 1117 em \% em massa

\begin{tabular}{|c|c|c|c|}
\hline $\mathrm{C}$ & $\mathrm{Mn}$ & $\mathrm{S}$ & $\mathrm{P}$ \\
\hline $0,14-0,20$ & $1,00-1,30$ & $0,08-0,13$ & 0,040 máx. \\
\hline
\end{tabular}

\subsection{Ensaios em Laboratório}

Foram realizados cinco ensaios para o aço SAE 1141, sendo quatro deles analisados no presente trabalho, ensaios B1, B2, B3 e B5, de composição semelhante, em forno elétrico resistivo. Para o aço SAE 1117 foram realizados 5 ensaios, sendo 3 deles citados neste trabalho, ensaios J2, J3 e J5.

Os experimentos ocorreram em forno elétrico resistivo modelo High Temperature Graphite Vac-Gas Furnace / HT 2100 Vac Special, fornecido pelo Fabricante Linn High Therm $\mathrm{GmbH}$, Alemanha. Foram realizados sob atmosfera de argônio, a uma taxa de aquecimento de $5^{\circ} \mathrm{C} / \mathrm{min}$.

A carga é constituída da mistura de ferro de alta pureza com as ligas usadas industrialmente na produção dos respectivos aços, vide Tabelas 3 e 4 . A carga total de cada ensaio tem como massa $1 \mathrm{~kg}$, a fusão foi realizada em cadinhos de alumina com $99 \%$ de pureza.

Tabela 3. Ligas utilizadas nos experimentos do aço SAE 1141 para $1 \mathrm{~kg}$ de carga, em gramas.

\begin{tabular}{|c|c|c|c|c|c|c|}
\hline Mn eletr & Grafita & Enxofre & $\mathrm{Fe}_{2} \mathrm{O}_{3}$ & $\mathrm{FeCrBC}$ & Ni eletr & Si puro \\
\hline 15,5 & 5,00 & 1,01 & 0,075 & 2,45 & 0,44 & 1,1 \\
\hline
\end{tabular}

Tabela 4. Ligas utilizadas nos experimentos do aço SAE 1117 para $1 \mathrm{~kg}$ de carga, em gramas.

\begin{tabular}{|c|c|c|c|c|}
\hline Mn puro & Grafita & Enxofre & $\mathrm{Fe}_{2} \mathrm{O}_{3}$ & Si puro \\
\hline 10,00 & 2,50 & 0,70 & 0,046 & 0,40 \\
\hline
\end{tabular}

Os autores testaram duas disposições de ensaio, chamadas de T1 e T2, vide Figuras 1 e 2. A disposição de ensaio referente ao aço SAE 1117 será chamada de T3, vide figura 3, conforme Kirichenco [5].

\footnotetext{
* Contribuição técnica ao $69^{\circ}$ Congresso Anual da ABM - Internacional e ao 14ํㅡㄹ ENEMET - Encontro Nacional de Estudantes de Engenharia Metalúrgica, de Materiais e de Minas, 21 a 25 de julho de 2014, São Paulo, SP, Brasil.
} 


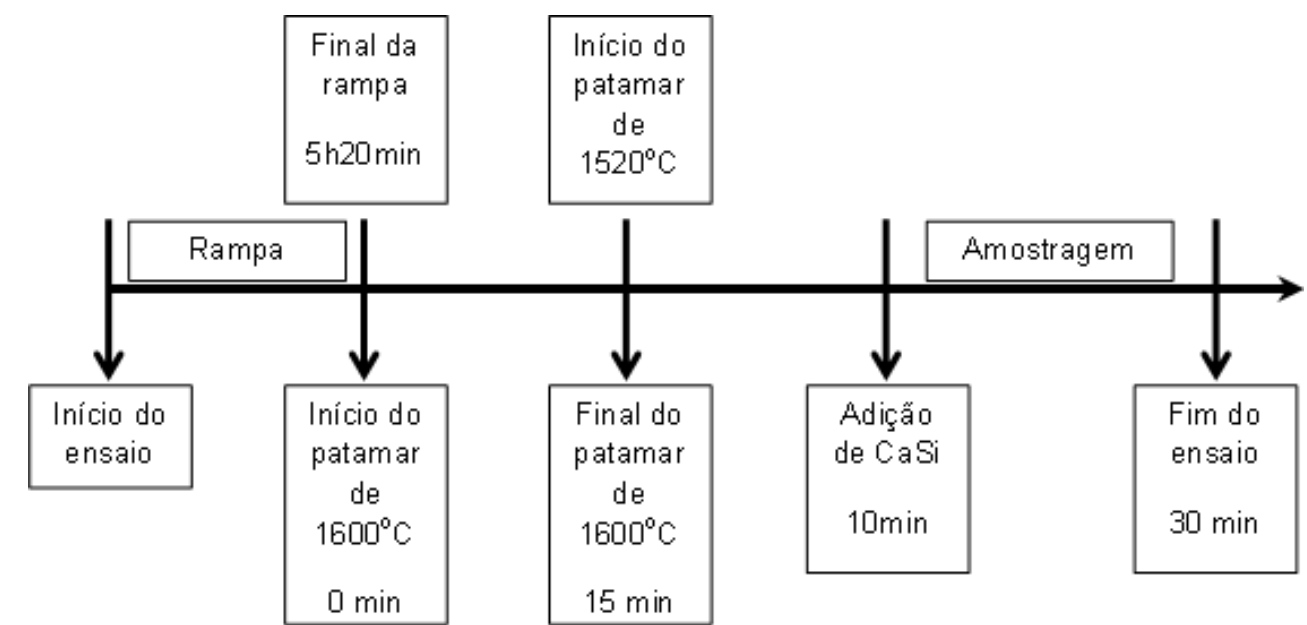

Figura 1. Fluxo do processo para a condição experimental T1.

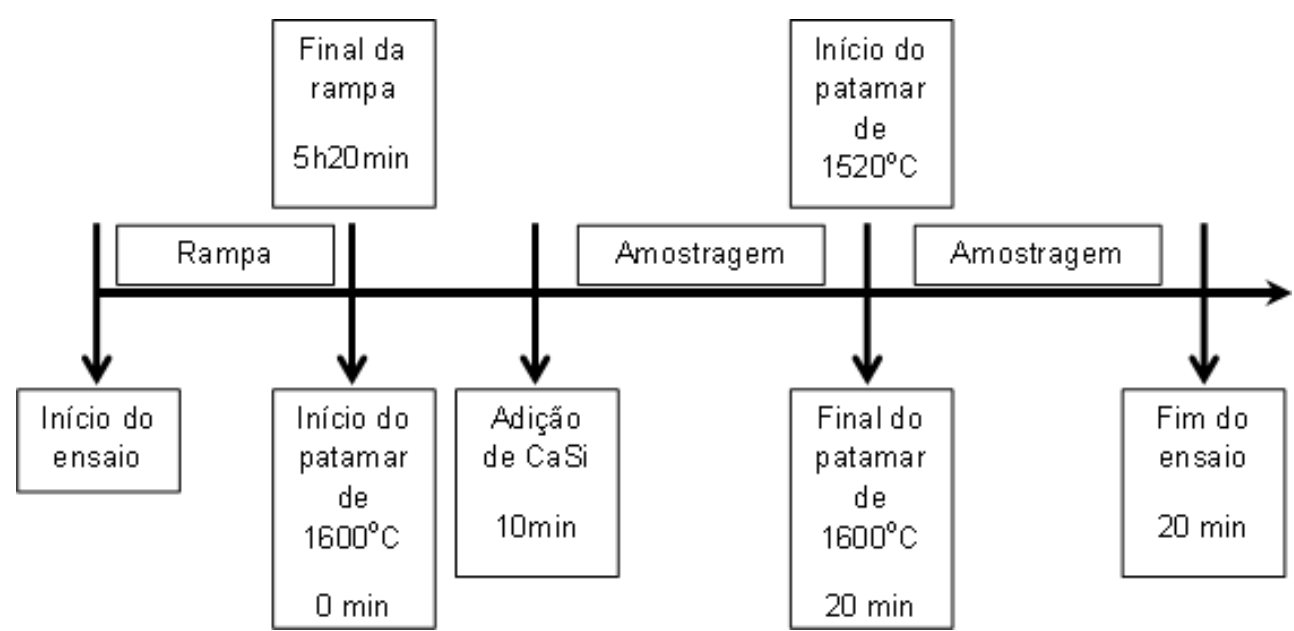

Figura 2. Fluxo do processo para a condição experimental T2.

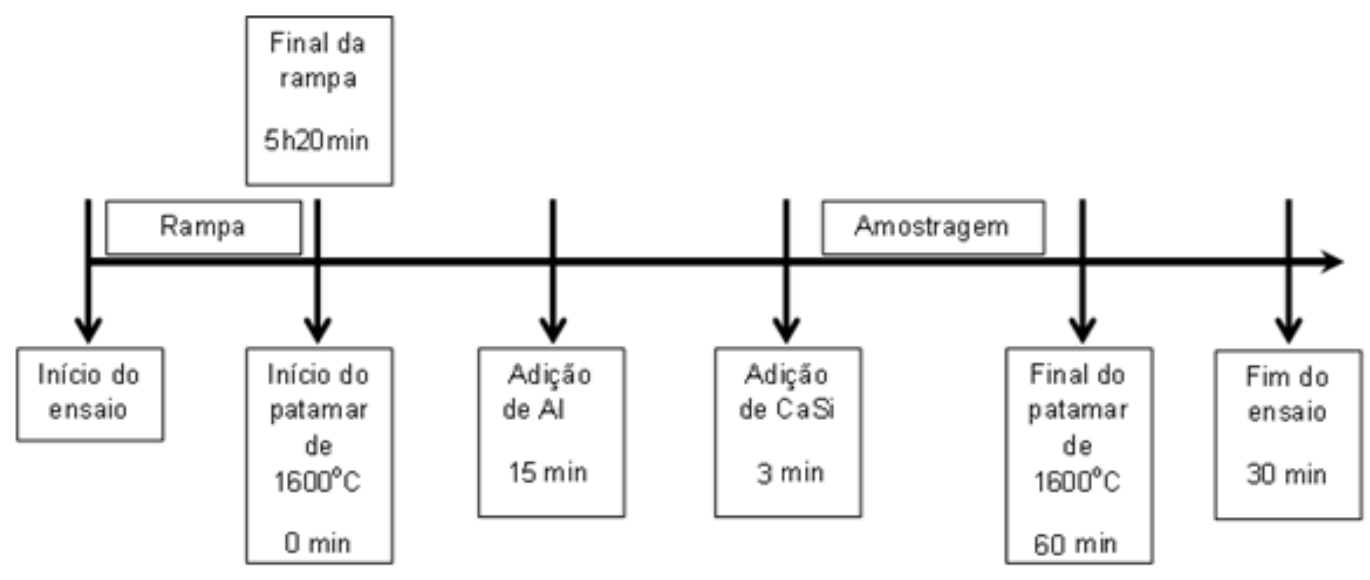

Figura 3. Fluxo do processo para a condição experimental T3. 


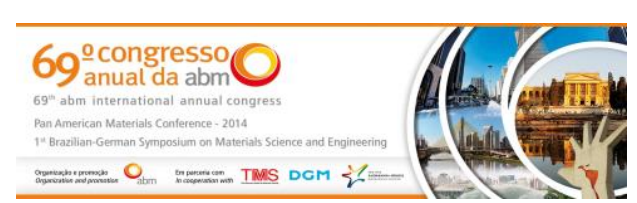

A Tabela 5 mostra a relação de ensaios realizados e condições de adição de CaSi e temperatura.

Tabela 5. Relação de ensaios realizados e suas condições.

\begin{tabular}{|c|c|c|c|c|}
\cline { 3 - 5 } \multicolumn{2}{c|}{} & $\begin{array}{c}\text { Condição } \\
\text { experimental }\end{array}$ & $\begin{array}{c}\text { Massa de CaSi } \\
\text { adicionada (g) }\end{array}$ & $\begin{array}{c}\text { Temperatura de } \\
\text { adição de CaSi }\end{array}$ \\
\hline \multirow{4}{*}{ SAE 1141 } & B1 & T1 & 0,95 & $1520^{\circ} \mathrm{C}$ \\
\cline { 2 - 5 } & B2 & T1 & 0,95 & $1520^{\circ} \mathrm{C}$ \\
\cline { 2 - 5 } & B3 & T2 & 0,95 & $1600^{\circ} \mathrm{C}$ \\
\cline { 2 - 5 } & B5 & T2 & 0,68 & $1600^{\circ} \mathrm{C}$ \\
\hline \multirow{3}{*}{ SAE 1117 } & J2 & T3 & 0,71 & $1600^{\circ} \mathrm{C}$ \\
\cline { 2 - 5 } & J3 & T3 & 0,71 & $1600^{\circ} \mathrm{C}$ \\
\cline { 2 - 5 } & J5 & T3 & 0,71 & $1600^{\circ} \mathrm{C}$ \\
\hline
\end{tabular}

\subsection{Preparação e Análise das Amostras}

As amostras coletadas ao longo do tempo dos ensaios - conforme exposto nas Figuras 1, 2 e 3 - foram cortadas em pinos de aproximadamente 1,5 cm, embutidas e submetidas à preparação metalográfica convencional. Posteriormente foram analisadas via Microscópio Eletrônico de Varredura (MEV/EDS), itens 2.2.1 e 2.2.2. As amostras de aço remanescente no cadinho passaram por análise química, item 2.2.3.

\subsubsection{Análise via MEV/EDS de forma manual}

As amostras dos pinos foram analisadas em Microscópio Eletrônico de Varredura (MEV/EDS) marca JEOL modelo 5800, no Centro de Microscopia Eletrônica da UFRGS.

Foram analisados quatro pinos dos ensaios B1 e B5 e três do ensaio B2 e B3 porque houve problema de preenchimento nas demais amostras, impedindo as análises. Foram estudados quatro pinos de cada amostra referente ao aço SAE 1117.

É relevante relatar que, após algumas tentativas, os autores concluíram ser vantajoso, para análise das amostras em questão, o uso do MEV em Elétrons Retroespalhados e tensões de 15 e 20 kV. É importante, para obter uma análise satisfatória, que haja um equilíbrio da tensão e dos elétrons emitidos utilizados para obtenção da imagem.

\subsubsection{Análise em microscópio automatizado}

As amostras de aço remanescente B1, B2, B3 e B5 foram ainda analisadas em microscópio eletrônico de varredura automatizado, ASPEX-PSEM explorer, acoplado a uma sonda de espectrometria de energia dispersiva (EDS).

Tal equipamento torna possível determinar a posição, quantidade, tamanho, morfologia e a composição química de cada inclusão não metálica encontrada na área analisada. A partir das composições químicas elementares das inclusões, uma série de critérios ou filtros identificam cada inclusão detectada.

Os resultados da composição química elementar das inclusões nas amostras, foram transferidos para uma planilha Excel para serem convertidos na forma de óxidos, o que, após sua normalização, foram plotados no sistema de diagrama ternário específico, $\mathrm{CaO}-\mathrm{SiO}_{2}-\mathrm{Al}_{2} \mathrm{O}_{3}$.

\subsubsection{Amostras para análise química}

\footnotetext{
* Contribuição técnica ao $69^{\circ}$ Congresso Anual da ABM - Internacional e ao 14ํㅡㄹ ENEMET - Encontro Nacional de Estudantes de Engenharia Metalúrgica, de Materiais e de Minas, 21 a 25 de julho de 2014, São Paulo, SP, Brasil.
} 


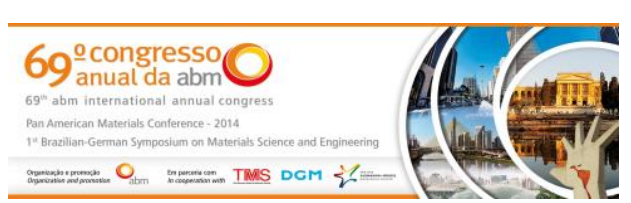

As amostras finais de aço de todos os ensaios foram analisadas no espectrômetro de emissão ótica modelo ARL 4460, sendo analisados 6 pontos por ensaio, a fim de se verificar se a composição ensaiada estava de acordo com o especificado para o aço estudado. A análise de oxigênio total foi realizada em duas amostras por ensaio, no Determinador Simultâneo de Nitrogênio e Oxigênio, marca LECO modelo 436.

\subsubsection{Simulação termodinâmica computacional}

A simulação termodinâmica computacional foi realizada através do software comercial FactSage, versão 6.4. Foi feito uso de dois bancos de dados, um para aço líquido (FTmisc-FeLQ) e o outro para as inclusões de óxidos e sulfetos (FToxid-SLAGA). Foi utilizado o módulo Equilib, módulo de minimização da energia livre de Gibbs.

Os dados de entrada foram: os resultados das análises das amostras de aço feitas no Espectrômetro de Emissão ótica e Determinador Simultâneo de Nitrogênio e Oxigênio na temperatura de $1600^{\circ} \mathrm{C}$, para representar a condição em uma panela siderúrgica.

\section{RESULTADOS E DISCUSSÃO}

Nesta seção serão discutidos os resultados obtidos nos experimentos realizados no forno elétrico resistivo na produção dos aços SAE 1141 e SAE 1117.

\subsection{Composição Química dos Aços}

Os resultados da análise química do aço SAE 1141 remanescente no cadinho estão demonstrados na tabela 6 , já na tabela 7 encontram-se os resultados da análise química referente às amostras do aço SAE 1117.

São objetivos da análise de composição química das amostras remanescentes do cadinho verificar se há completa homogeneização química e se os experimentos foram eficientes em reproduzir amostras na faixa de composição designada para os aços estudados.

Na Figura 4, é mostrada a "fatia" de aço e a distribuição dos pontos onde é feita a análise química.

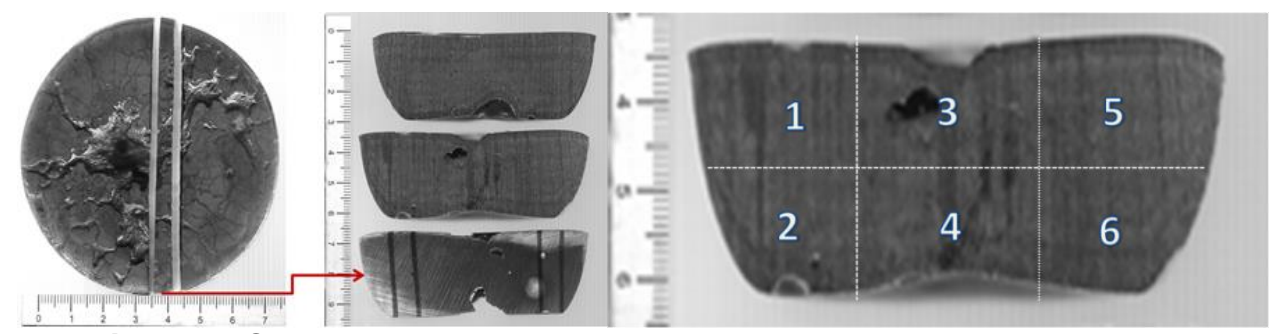

Figura 4. Obtenção da bolacha de aço e o produto do corte transversal.

Tabela 6. Resultados das análises químicas do aço (base SAE 1141) em \% em massa.

\begin{tabular}{|c|c|c|c|c|c|c|c|c|c|c|c|c|}
\hline B1 & $\mathrm{C}$ & $\mathrm{Si}$ & $\mathrm{Mn}$ & $\mathrm{P}$ & $\mathrm{S}$ & $\mathrm{Cr}$ & $\mathrm{Ni}$ & $\mathrm{Mo}$ & $\mathrm{Al}^{*}$ & $\mathrm{Ca}^{*}$ & $\mathrm{O}_{\text {total }}{ }^{*}$ & $\mathrm{~N}^{*}$ \\
\hline Média & 0,38 & 0,18 & 1,53 & 0,01 & 0,11 & 0,20 & 0,07 & 0,01 & 20,00 & 10,00 & 27,50 & 4,5 \\
\hline
\end{tabular}

\footnotetext{
* Contribuição técnica ao 69 Congresso Anual da ABM - Internacional e ao 14 ENEMET - Encontro Nacional de Estudantes de Engenharia Metalúrgica, de Materiais e de Minas, 21 a 25 de julho de 2014, São Paulo, SP, Brasil.
} 


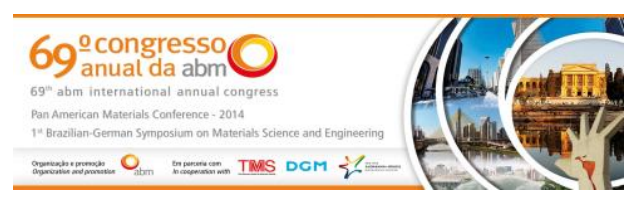

\begin{tabular}{|l|l|l|l|l|l|l|l|l|l|l|l|l|} 
Desvio & 0 & 0 & 0,01 & 0 & 0,01 & 0,01 & 0 & 0 & 0 & 2,61 & 7,78 & 0,7 \\
\hline
\end{tabular}

\begin{tabular}{|c|c|c|c|c|c|c|c|c|c|c|c|c|}
\hline B2 & $\mathrm{C}$ & $\mathrm{Si}$ & $\mathrm{Mn}$ & $\mathrm{P}$ & $\mathrm{S}$ & $\mathrm{Cr}$ & $\mathrm{Ni}$ & $\mathrm{Mo}$ & $\mathrm{Al}^{*}$ & $\mathrm{Ca}^{*}$ & $\mathrm{O}_{\text {total }}$ & $\mathrm{N}^{*}$ \\
\hline Média & 0,37 & 0,21 & 1,39 & 0,02 & 0,09 & 0,19 & 0,07 & 0,01 & 41,67 & 13,83 & 33,50 & 4,0 \\
\hline Desvio & 0 & 0 & 0,08 & 0 & 0,01 & 0 & 0 & 0 & 4,08 & 1,72 & 2,12 & 0 \\
\hline
\end{tabular}

\begin{tabular}{|c|c|c|c|c|c|c|c|c|c|c|c|c|}
\hline B3 & $\mathrm{C}$ & $\mathrm{Si}$ & $\mathrm{Mn}$ & $\mathrm{P}$ & $\mathrm{S}$ & $\mathrm{Cr}$ & $\mathrm{Ni}$ & $\mathrm{Mo}$ & $\mathrm{Al}^{*}$ & $\mathrm{Ca}^{*}$ & $\mathrm{O}_{\text {total }}{ }^{*}$ & $\mathrm{~N}^{*}$ \\
\hline Média & 0,38 & 0,21 & 1,56 & 0,01 & 0,09 & 0,19 & 0,07 & 0,01 & 46,70 & 19,80 & 42,00 & 4,0 \\
\hline Desvio & 0,01 & 0 & 0,06 & 0 & 0,02 & 0 & 0 & 0 & 5,16 & 3,27 & 2,00 & 0 \\
\hline
\end{tabular}

\begin{tabular}{|c|c|c|c|c|c|c|c|c|c|c|c|c|}
\hline B5 & $\mathrm{C}$ & $\mathrm{Si}$ & $\mathrm{Mn}$ & $\mathrm{P}$ & $\mathrm{S}$ & $\mathrm{Cr}$ & $\mathrm{Ni}$ & $\mathrm{Mo}$ & $\mathrm{Al}^{*}$ & $\mathrm{Ca}^{*}$ & $\mathrm{O}_{\text {total }}$ & $\mathrm{N}^{*}$ \\
\hline Média & 0,40 & 0,18 & 1,50 & 0,01 & 0,10 & 0,20 & 0,07 & 0,01 & 32,00 & 16,75 & 39,00 & 6,0 \\
\hline Desvio & 0 & 0 & 0,02 & 0 & 0,03 & 0 & 0 & 0 & 4,47 & 1,26 & 1,41 & 0 \\
\hline
\end{tabular}
*em ppm

Tabela 7. Resultados das análises química do aço (base SAE 1117) em \% em massa.

\begin{tabular}{|c|c|c|c|c|c|c|c|c|c|c|c|c|}
\hline J2 & $\mathrm{C}$ & $\mathrm{Si}$ & $\mathrm{Mn}$ & $\mathrm{P}$ & $\mathrm{S}$ & $\mathrm{Cr}$ & $\mathrm{Ni}$ & $\mathrm{Mo}$ & $\mathrm{Al}^{*}$ & $\mathrm{Ca}^{*}$ & $\mathrm{O}_{\text {total }}{ }^{*}$ & $\mathrm{~N}^{*}$ \\
\hline Média & 0,20 & 0,10 & 0,79 & 0,01 & 0,06 & 0,10 & 0,04 & 0,01 & 22,08 & 10,27 & 21,10 & 2,5 \\
\hline Desvio & 0,22 & 0,11 & 0,86 & 0,01 & 0,04 & 0,11 & 0,04 & 0,01 & 20,82 & 9,36 & 22,43 & 3 \\
\hline
\end{tabular}

\begin{tabular}{|c|c|c|c|c|c|c|c|c|c|c|c|c|}
\hline J3 & $\mathrm{C}$ & $\mathrm{Si}$ & $\mathrm{Mn}$ & $\mathrm{P}$ & $\mathrm{S}$ & $\mathrm{Cr}$ & $\mathrm{Ni}$ & $\mathrm{Mo}$ & $\mathrm{Al}^{*}$ & $\mathrm{Ca}^{*}$ & $\mathrm{O}_{\text {total }^{*}}$ & $\mathrm{~N}^{*}$ \\
\hline Média & 0,20 & 0,10 & 0,79 & 0,01 & 0,06 & 0,10 & 0,04 & 0,01 & 19,84 & 9,41 & 20,99 & 2,88 \\
\hline Desvio & 0,16 & 0,07 & 0,61 & 0 & 0,03 & 0,08 & 0,03 & 0 & 11,40 & 6,35 & 0 & 0 \\
\hline
\end{tabular}

\begin{tabular}{|c|c|c|c|c|c|c|c|c|c|c|c|c|}
\hline J5 & C & $\mathrm{Si}$ & $\mathrm{Mn}$ & $P$ & $S$ & $\mathrm{Cr}$ & $\mathrm{Ni}$ & Mo & $\overline{A l^{*}}$ & $\mathrm{Ca}^{*}$ & $\mathrm{O}_{\text {total }}{ }^{*}$ & $\mathrm{~N}^{*}$ \\
\hline Média & 0,20 & 0,10 & 0,76 & 0,01 & 0,05 & 0,10 & 0,04 & 0,01 & 18,54 & 8,85 & 16,13 & 2,09 \\
\hline Desvio & 0,02 & 0,02 & 0,11 & 0 & 0,01 & 0,01 & 0 & 0 & 4,84 & 1,72 & 10,77 & 1,41 \\
\hline
\end{tabular}

Todos os elementos principais ( $\mathrm{C}$ até $\mathrm{Mo}$ ) das tabelas apresentaram baixo desviopadrão, o que era desejado para ambos os aços.

Entre os ensaios submetidos à adição de $0,95 \mathrm{~g}$ de $\mathrm{CaSi}$ (ensaios B1, B2 e B3), observa-se que o terceiro apresenta teor mais elevado de Ca na sua composição química em relação aos demais, devido ao fato de a liga ter sido adicionado em temperatura mais elevada.

Comparando os ensaios B3, B5, J2, J3 e J5 - todos com adição de CaSi em $1600^{\circ} \mathrm{C}$ o ensaio B3 obteve um maior rendimento de $\mathrm{Ca}$ em relação aos demais. Todavia seu desvio padrão é mais elevado relação à amostra B5, evidenciando o maior controle de homogeneização quando adicionado menor quantidade de liga.

Observa-se também que nos ensaios B1 e B2 teor de Ca é inferior a média de 20 ppm esperado para experimentos com tal teor de liga CaSi adicionada.

A Tabela 8 mostra o teor de Al e Ca em cada ponto dos aços ensaiados.

Tabela 8. Teor de Al e Ca dos ensaios B1 e B2 em ppm.

\footnotetext{
* Contribuição técnica ao $69^{\circ}$ Congresso Anual da ABM - Internacional e ao 14ํㅡㄹ ENEMET - Encontro Nacional de Estudantes de Engenharia Metalúrgica, de Materiais e de Minas, 21 a 25 de julho de 2014, São Paulo, SP, Brasil.
} 


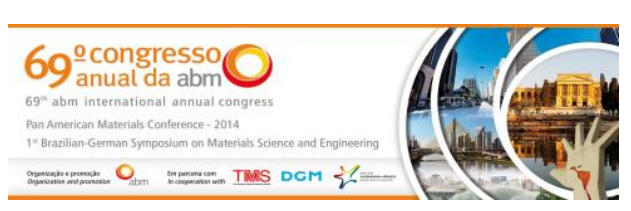

\begin{tabular}{|c|c|c|c|c|}
\hline & \multicolumn{2}{|c|}{$\mathrm{B} 1$} & \multicolumn{2}{c|}{ B2 } \\
\hline Ponto & $\mathrm{Al}$ & $\mathrm{Ca}$ & $\mathrm{Al}$ & $\mathrm{Ca}$ \\
\hline 1 & 20 & 7 & 40 & 12 \\
\hline 2 & 20 & 8 & 40 & 16 \\
\hline 3 & 20 & 14 & 40 & 15 \\
\hline 4 & 20 & 12 & 40 & 12 \\
\hline 5 & 20 & 9 & 40 & 13 \\
\hline 6 & 20 & 10 & 50 & 15 \\
\hline
\end{tabular}

Observa-se que os pontos 3 e 4, de localização central onde a liga é injetada, possuem teor de cálcio mais elevado em relação aos demais. Há duas hipóteses possíveis: a dissolução não uniforme da liga de CaSi e a presença de porosidades no centro da amostra, o que pode gerar resultados incorretos de análise.

\subsection{Análise de Oxigênio Total}

Conforme Zhang e Thomas [6], a medição do oxigênio serve como uma medida direta de avaliação de limpeza de aços. Larsen e Fruehan [3] também afirmam que, durante o processo na aciaria, é possível determinar a extensão da modificação das inclusões pela medição da atividade do oxigênio através do uso de equipamento apropriado.

O oxigênio total do aço consiste na soma do oxigênio dissolvido e o combinado na forma de óxidos. Subtraindo o teor de oxigênio dissolvido do valor do oxigênio total é viável inferir o nível de inclusões no aço.

Tabela 9. Análise de oxigênio nos ensaios dos aços SAE 1117 e SAE 1141.

\begin{tabular}{|c|c|c|c|c|}
\cline { 2 - 5 } \multicolumn{1}{c|}{} & \multicolumn{2}{c|}{ Ototal } & \multirow{2}{*}{ Odissolvido } & \multirow{2}{*}{ Oinclusões } \\
\cline { 2 - 5 } & Média & Desvio & & \\
\hline B1 & 27,50 & 7,78 & 13,99 & 13,51 \\
\hline B2 & 33,50 & 2,12 & 11,29 & 22,21 \\
\hline B3 & 42,00 & 2,00 & 10,92 & 31,08 \\
\hline B5 & 39,00 & 1,41 & 13,35 & 25,65 \\
\hline J2 & 21,10 & 22,43 & 10,69 & 10,41 \\
\hline J3 & 20,99 & 0,00 & 11,38 & 9,60 \\
\hline J5 & 16,13 & 10,77 & 9,26 & 6,87 \\
\hline
\end{tabular}

$\mathrm{Na}$ Tabela 9 encontra-se o Ototal resultante da medida com espectrômetro de emissão ótica, o Odissolvido estimado através de simulação termodinâmica e o Oinclusões estimado como a diferença entre os dois primeiros. Observa-se que as amostras J2, J3 e J5 apresentam Oinclusões inferior as demais amostras. Tal resultado é esperado, tendo em vista que esses experimentos receberam teor mais elevado de Al em sua preparação, possuindo uma condição mais favorável de desoxidação.

\footnotetext{
* Contribuição técnica ao $69^{\circ}$ Congresso Anual da ABM - Internacional e ao 14ํㅡㄹ ENEMET - Encontro Nacional de Estudantes de Engenharia Metalúrgica, de Materiais e de Minas, 21 a 25 de julho de 2014, São Paulo, SP, Brasil.
} 


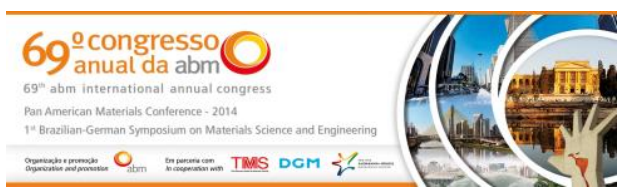

\subsection{Análise de Inclusões do Aço Remanescente Através de Microscópio Automatizado}

As amostras de aço remanescente B1, B2, B3 e B5 foram analisadas através de microscópio automatizado (ASPEX), conforme descrito na metodologia do trabalho e comparadas aos resultados obtidos via simulação termodinâmica, vide tabela 10 .

Tabela 10. Resultados da análise de inclusões via ASPEX e simulação termodinâmica.

\begin{tabular}{|c|c|c|c|c|c|c|c|c|c|}
\cline { 2 - 10 } \multicolumn{1}{c|}{} & \multicolumn{3}{c|}{$\mathrm{CaO}(\%)$} & \multicolumn{3}{c|}{$\mathrm{SiO}_{2}(\%)$} & \multicolumn{3}{c|}{$\mathrm{Al}_{2} \mathrm{O}_{3}(\%)$} \\
\cline { 2 - 10 } & Média & Desvio & Simulação & Média & Desvio & Simulação & Média & Desvio & Simulação \\
\hline B1 & 29,19 & 20,77 & 41,20 & 33,51 & 21,61 & 28,27 & 37,30 & 28,89 & 30,53 \\
\hline B2 & 48,31 & 15,97 & 35,51 & 16,58 & 14,89 & 19,91 & 35,10 & 13,80 & 44,58 \\
\hline B3 & 43,13 & 6,92 & 36,53 & 11,78 & 2,60 & 20,27 & 45,08 & 8,30 & 43,20 \\
\hline B5 & 38,82 & 23,27 & 37,72 & 31,20 & 21,91 & 24,48 & 29,98 & 23,83 & 37,79 \\
\hline
\end{tabular}

Os resultados das amostras B1 e B2 são bastante distintos em relação às amostras retiradas durante esses ensaios, evidenciando novamente a dificuldade de homogeneização de tal quantidade de liga na configuração de ensaio T1. Observando os aços B3 e B5 constata-se que em ambos foi identificado um percentual de $\mathrm{CaO}$ superior a $35 \%$ nas inclusões, evidenciando que há formação de cálcio-aluminatos líquidos.

A composição das inclusões analisadas via ASPEX correspondem satisfatoriamente com os resultados obtidos através de simulação termodinâmica.

Segundo Kirichenco [5], os cálcio-aluminatos líquidos adquirem formato globular no banho e o mantém durante a solidificação e na conformação. Observou-se no presente artigo tais inclusões de modo satisfatório, vide figura 5.

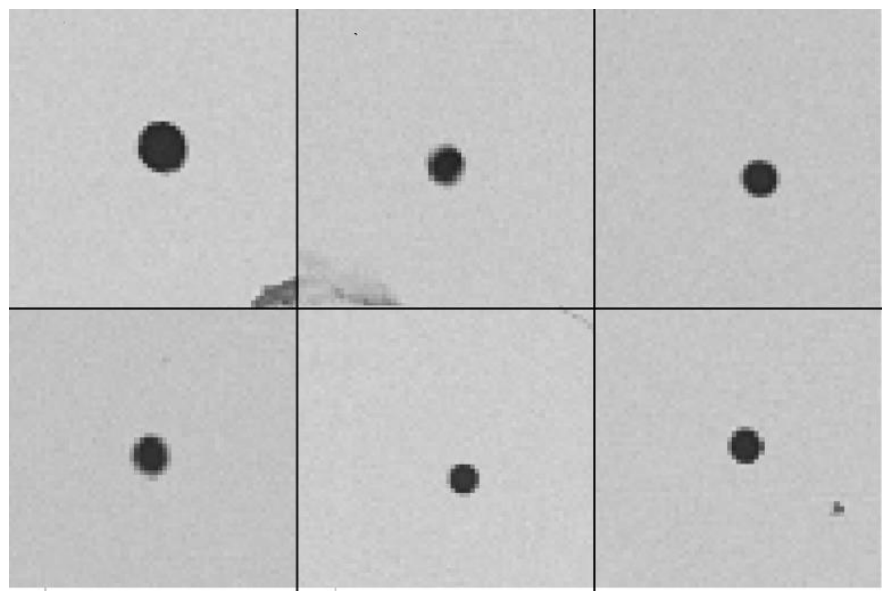

Figura 5. Inclusões de cálcio-aluminatos analisadas via ASPEX.

Ainda foram observadas inclusões ricas em sulfetos, vide figura 6 . Era esperada a presença dessas inclusões no aço remanescente e não nos pinos coletados, porque o enxofre é solúvel no aço fundido, mas no aço sólido sua solubilização é muito lenta. Ele é precipitado sob a forma de sulfetos de cálcio (CaS) e manganês (MnS) durante a solidificação e o padrão de precipitação é influenciado pela segregação, conforme Kiessling [7].

\footnotetext{
* Contribuição técnica ao $69^{\circ}$ Congresso Anual da ABM - Internacional e ao 14ํㅡㄹ ENEMET - Encontro Nacional de Estudantes de Engenharia Metalúrgica, de Materiais e de Minas, 21 a 25 de julho de 2014, São Paulo, SP, Brasil.
} 

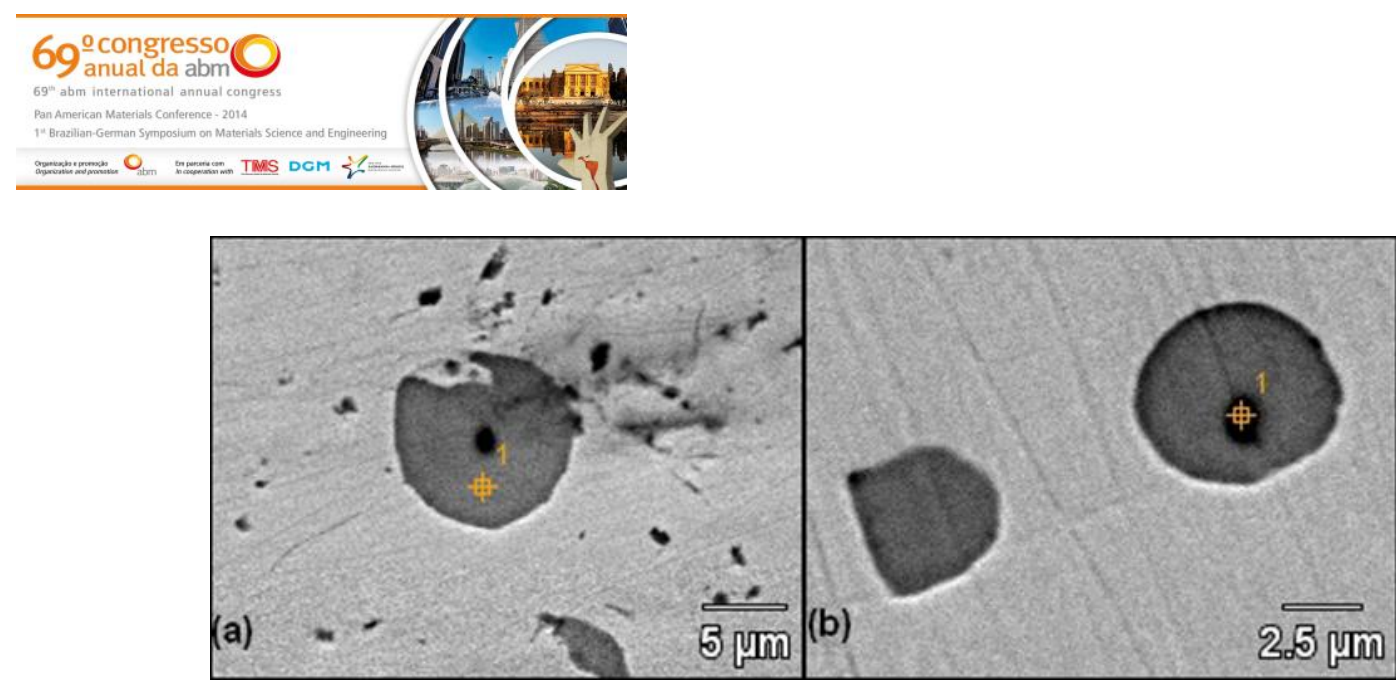

Figura 6. Inclusões de MnS analisadas via ASPEX.

A presença de enxofre pode estar associada aos sulfetos de CaS e/ou MnS nas inclusões. De acordo com Cicutti et al [8], a formação dos sulfetos de cálcio é influenciada pela composição do aço (enxofre, cálcio, alumínio e oxigênio total) e pela temperatura e tais sulfetos, conforme Madias [9], ocorrendo na panela e/ou no distribuidor, pode ocasionar o entupimento da válvula. Ao acontecer no molde, podem causar rompimento do veio. No caso de ocorrência na solidificação do aço, se observam isolados ou ao redor dos óxidos.

\subsection{Análise de Inclusões Através de MEV/EDS de Forma Manual}

Na Tabela 11 observa-se o resultado total da análise das inclusões, média e desvio padrão, obtida nos pinos retirados ao longo dos ensaios.

Tabela 11. Resultado da análise de inclusões via MEV/EDS.

\begin{tabular}{|c|c|c|c|c|c|c|}
\hline \multicolumn{6}{|c|}{ Amostra $\mathrm{B} 3$ - Geral (20 inclusões) } & \multicolumn{4}{c|}{$\mathrm{CaO}$} & $\mathrm{MnO}$ \\
\hline & $\mathrm{MgO}$ & $\mathrm{Al}_{2} \mathrm{O}_{3}$ & $\mathrm{SiO}_{2}$ & $\mathrm{~S}$ & $\mathrm{CaO}$ \\
\hline Média & 5,10 & 32,04 & 17,66 & 2,70 & 31,27 & 11,24 \\
\hline Desvio & 4,47 & 12,34 & 7,57 & 1,94 & 7,60 & 6,92 \\
\hline
\end{tabular}

\begin{tabular}{|c|c|c|c|c|c|c|}
\hline \multicolumn{2}{|c|}{ Amostra B5 - Geral (32 inclusões) } & \multicolumn{1}{l|}{} \\
\hline & $\mathrm{MgO}$ & $\mathrm{Al}_{2} \mathrm{O}_{3}$ & $\mathrm{SiO}_{2}$ & $\mathrm{~S}$ & $\mathrm{CaO}$ & $\mathrm{MnO}$ \\
\hline Média & 1,09 & 24,78 & 29,79 & 2,39 & 34,72 & 7,23 \\
\hline Desvio & 0,93 & 8,17 & 3,50 & 1,68 & 6,91 & 3,07 \\
\hline
\end{tabular}

\begin{tabular}{|c|c|c|c|c|c|c|}
\hline \multicolumn{7}{|c|}{ Amostra J2 - Geral (53 inclusões) } \\
\cline { 1 - 4 } & $\mathrm{MgO}$ & $\mathrm{Al}_{2} \mathrm{O}_{3}$ & $\mathrm{SiO}_{2}$ & $\mathrm{~S}$ & $\mathrm{CaO}$ & $\mathrm{MnO}$ \\
\hline Média & 1,22 & 56,11 & 0,67 & 14,12 & 9,79 & 18,1 \\
\hline Desvio & 0,08 & 8,35 & 0,44 & 5,03 & 0,86 & 8,8 \\
\hline
\end{tabular}

Amostra J3 - Geral (54 inclusões)

\begin{tabular}{|c|c|c|c|c|c|c|}
\hline & $\mathrm{MgO}$ & $\mathrm{Al}_{2} \mathrm{O}_{3}$ & $\mathrm{SiO}_{2}$ & $\mathrm{~S}$ & $\mathrm{CaO}$ & $\mathrm{MnO}$ \\
\hline Média & 1,86 & 75,28 & 2,10 & 7,06 & 9,35 & 4,35 \\
\hline Desvio & 0,34 & 6,31 & 0,80 & 3,89 & 2,88 & 6,95 \\
\hline
\end{tabular}

\begin{tabular}{|c|c|c|c|c|c|c|}
\hline \multicolumn{2}{|c|}{ Amostra J5 - Geral (63 inclusões) } & \multicolumn{1}{l|}{} \\
\hline & $\mathrm{MgO}$ & $\mathrm{Al}_{2} \mathrm{O}_{3}$ & $\mathrm{SiO}_{2}$ & $\mathrm{~S}$ & $\mathrm{CaO}$ & $\mathrm{MnO}$ \\
\hline Média & 1,16 & 64,21 & 3,38 & 7,84 & 8,67 & 14,73 \\
\hline Desvio & 0,20 & 2,94 & 2,18 & 3,73 & 0,75 & 5,51 \\
\hline
\end{tabular}

\footnotetext{
* Contribuição técnica ao $69^{\circ}$ Congresso Anual da ABM - Internacional e ao 14ํㅡㄹ ENEMET - Encontro Nacional de Estudantes de Engenharia Metalúrgica, de Materiais e de Minas, 21 a 25 de julho de 2014, São Paulo, SP, Brasil.
} 


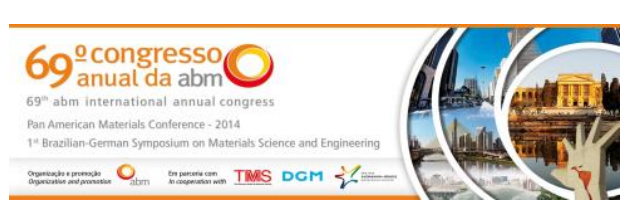

Nos ensaios B1 e B2, foram observados dois grupos de inclusões. O primeiro encontra-se no sistema $\mathrm{MnO}-\mathrm{Al}_{2} \mathrm{O}_{3}-\mathrm{SiO}_{2}$ com traços de $\mathrm{MgO}$ e $\mathrm{CaO}$ enquanto 0 segundo é composto por um percentual em massa de $\mathrm{CaO}$ superior a $80 \%$. Tais resultados evidenciam novamente que em ambos os ensaios, não ocorreu à homogeneização satisfatória da liga CaSi adicionada ao banho. Dessa forma o primeiro grupo de inclusões representa a desoxidação com $\mathrm{Mn}$ e Si, com Al residual, já o segundo grupo mostra que a liga de CaSi não se difundiu de forma adequada. $\mathrm{A}$ presença de traços de $\mathrm{MgO}$ se deve a presença de $\mathrm{Mg}$ residual nas ligas utilizadas.

Comparando os experimentos referentes ao aço SAE 1141, B3 e B5, observa-se que as inclusões estão no sistema $(\mathrm{CaO}, \mathrm{MnO})-\mathrm{Al}_{2} \mathrm{O}_{3}-\mathrm{SiO}_{2} . \mathrm{O} \mathrm{CaO}$ tende a substituir o MnO no produto de desoxidação conforme ocorre a reação entre o cálcio e as inclusões formadas. A proporção de $\mathrm{CaO}$ e $\mathrm{MnO}$ depende do grau de oxidação do banho. Menores teores de oxigênio total implicam em maiores teores de $\mathrm{CaO}$ nas inclusões. O oxigênio total da B5 foi ligeiramente menor do que na B3.

As amostras referentes ao SAE 1117 possuem inclusões com teor bastante elevado de $\mathrm{Al}_{2} \mathrm{O}_{3}$ em relação as demais, tal resultado se deve ao fato de que nesses ensaios houve adição de $\mathrm{Al}$ anteriormente a adição de $\mathrm{CaSi}$, vide figura 3 . Segundo Beskow et al. [10], a adição de alumínio não apenas resulta na formação de inclusões de alumina, mas também afeta o comportamento químico das inclusões. O modo e a taxa em que ocorre a nucleação da alumina possuem um grande impacto no processo de desoxidação.

\subsection{Evolução da Composição das Inclusões ao Longo do Tempo}

A modificação das inclusões pelo cálcio é rápida, o que está de acordo com a literatura, ainda que os mecanismos não sejam plenamente conhecidos.

Lu et al [11], em um estudo sobre a cinética de modificação de inclusões com cálcio, observam que, em um minuto após a adição de cálcio, já não é mais possível verificar a presença de inclusões de alumina, havendo apenas cálcio-aluminatos presentes no banho.

Na Figura 7 se pode observar a evolução da composição química média das inclusões para o ensaio B3.

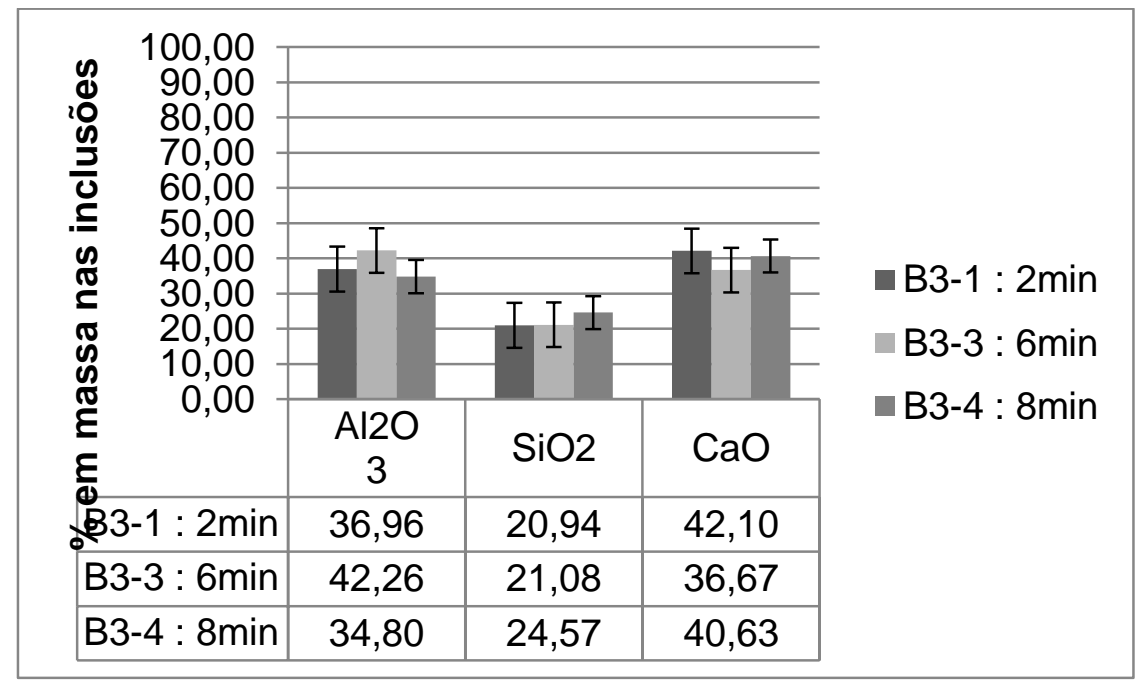

Figura 7. Evolução da composição química média das inclusões para o ensaio B3.

\footnotetext{
* Contribuição técnica ao $69^{\circ}$ Congresso Anual da ABM - Internacional e ao 14ํㅡㄹ ENEMET - Encontro Nacional de Estudantes de Engenharia Metalúrgica, de Materiais e de Minas, 21 a 25 de julho de 2014, São Paulo, SP, Brasil.
} 


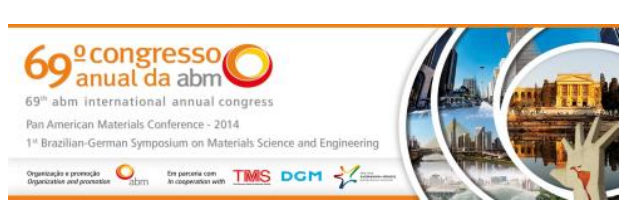

Sobre a Figura 7, as diferenças de composição entre (B3-1) e (B3-3; B3-4) podem ser devido às diferentes temperaturas (1600ㄷ para B3-1) e (1520ㄷ $\mathrm{C}$ para B3-3; B34) e tempo transcorrido após a adição de CaSi (B3-1 foi a primeira amostra retirada). Na Figura 8 se pode observar a evolução da composição química média das inclusões para o ensaio B5.

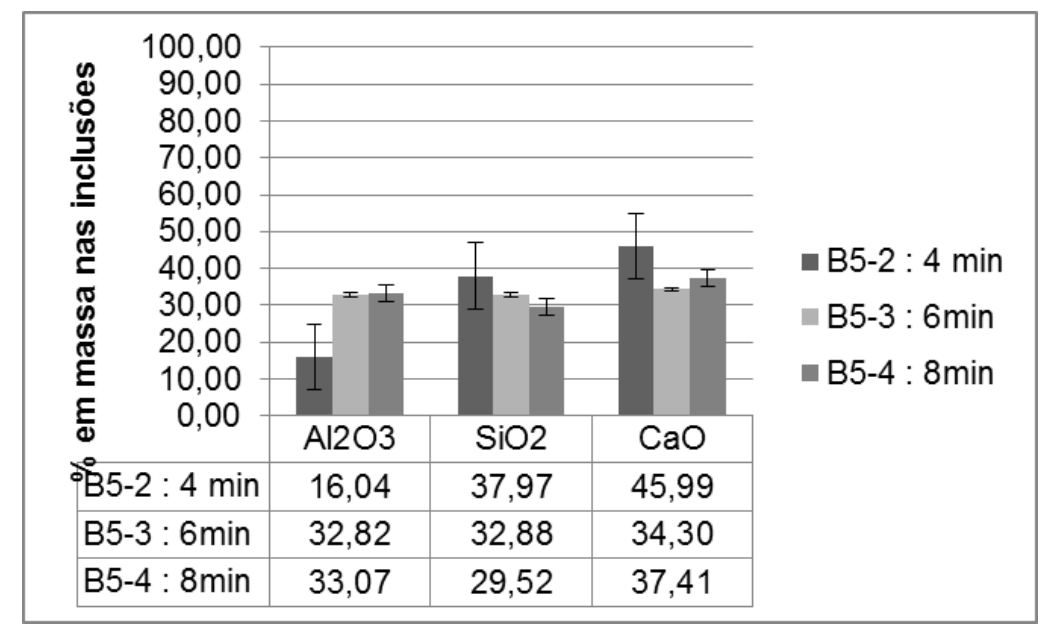

Figura 8. Evolução da composição química média das inclusões para o ensaio B5.

Analisando o ensaio $\mathrm{B} 5$, vide Figura 8, chama atenção a variação do teor de $\mathrm{Al}_{2} \mathrm{O}_{3}$ da amostra B5-2 em relação às demais amostras.

As amostras $\mathrm{B} 3$ e $\mathrm{B} 5$ apresentam inclusões no sistema $\mathrm{CaO}-\mathrm{Al}_{2} \mathrm{O}_{3}-\mathrm{SiO}_{2}$.

A evolução da composição das inclusões nas amostras J2, J3 e J5 é bastante semelhante, dessa forma nesse trabalho será apresentado o gráfico referente à amostra J3 (vide Figura 9).

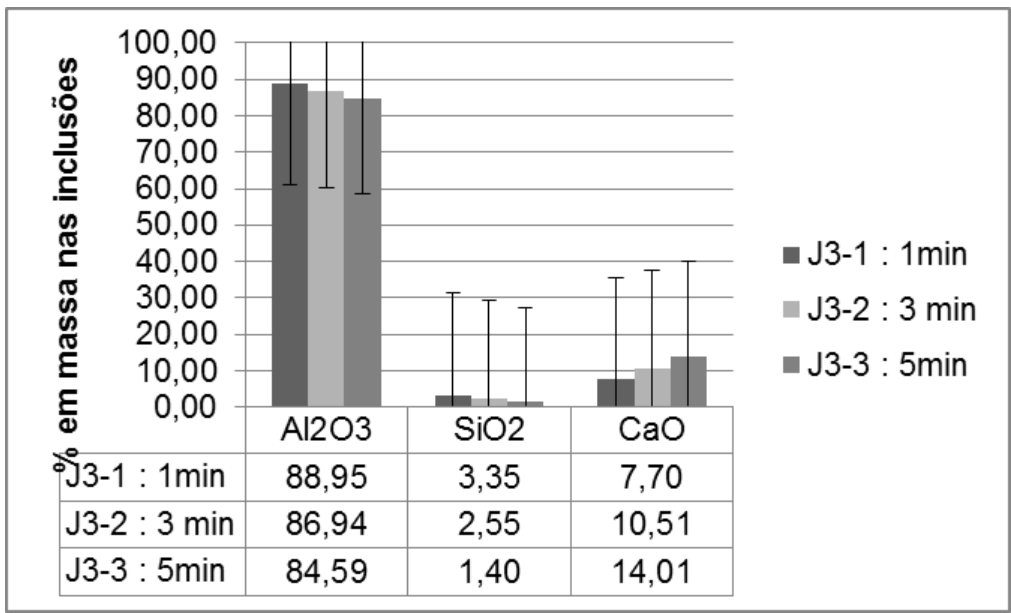

Figura 9. Evolução da composição química média das inclusões para o ensaio J3.

Observa-se que o teor de $\mathrm{SiO}_{2}$ apresentou a menor porcentagem em massa nas inclusões e $\mathrm{Al}_{2} \mathrm{O}_{3}$ a maior. Também se observa que há queda na porcentagem de $\mathrm{Al}_{2} \mathrm{O}_{3}$ e aumento na porcentagem de $\mathrm{CaO}$ ao longo do tempo. Tal comportamento difere do apresentado pelas amostras referentes ao aço SAE 1141 e pode estar ocorrendo devido à adição de alumínio ao ensaio. As amostras J2, J3 e J5 - conforme item 3.2 - apresentam Oinclusões inferior às demais amostras, devido à menor adição de $\mathrm{Fe}_{2} \mathrm{O}_{3}$ como fonte de oxigênio para o banho.

\footnotetext{
* Contribuição técnica ao 69ำ Congresso Anual da ABM - Internacional e ao 14ํㅡㄹ ENEMET - Encontro Nacional de Estudantes de Engenharia Metalúrgica, de Materiais e de Minas, 21 a 25 de julho de 2014, São Paulo, SP, Brasil.
} 


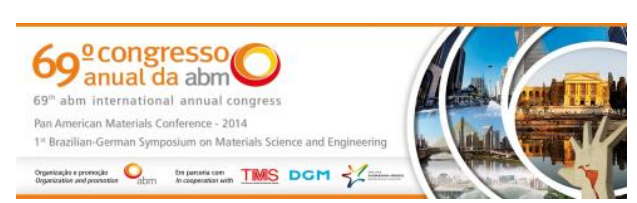

\section{CONCLUSÃo}

Neste estudo foi avaliada a modificação das inclusões com o tratamento com cálcio para os aços SAE 1141 e SAE 1117, em escala laboratorial. Os experimentos foram eficazes em reproduzir os aços em questão.

As inclusões nos ensaios $\mathrm{B} 1$ e $\mathrm{B} 2$ são extremamente ricas em $\mathrm{CaO}$ devido a não homogeneização da liga no banho. Assim, a disposição de temperatura T1 se mostrou ineficiente em experimentos com adição tão elevada de cálcio.

Os resultados dos ensaios realizados na disposição de temperatura T2 se mostraram mais satisfatórios que os realizados na disposição de temperatura T1 no que se refere à homogeneidade das amostras, harmonia com os dados apurados através da simulação termodinâmica e a morfologia globular das inclusões. Ainda no ensaio B5, houve maior correlação entre os resultados obtidos via MEV/EDS e a simulação termodinâmica. Tal constatação salienta a maior facilidade de controle de experimentos com adição inferior de cálcio.

O ensaio B3 apresentou teor mais elevado de Ca na sua composição química entre os experimentos com adição de $0,95 \mathrm{~g}$ de CaSi possivelmente devido ao fato de a liga ter sido realizado na disposição de temperatura $T 2$.

As amostras referentes ao aço SAE 1117 apresentaram Oinclusoees inferior às amostras referentes ao aço SAE 1141 devido ao fato de que as primeiras receberam menor quantidade de $\mathrm{Fe}_{2} \mathrm{O}_{3}$ em sua preparação.

Há significativa disparidade entre os resultados das amostras de aço remanescente (analisadas via ASPEX) e as amostras retiradas durante os ensaios B1 e B2 (analisadas via MEV). Tais diferenças são explicadas através de duas hipóteses, i) o fato de a análise via ASPEX ser mais eficiente em relação a analise via MEV/EDS (manual), ii) a dificuldade de homogeneização de tal quantidade de liga na configuração de ensaio T1.

Houve formação satisfatória de inclusões líquidas nos ensaios B3 e B5, evidenciando a eficiência da configuração de temperatura T2.

As amostras referentes ao SAE 1117 possuem inclusões com teor bastante elevado de $\mathrm{Al}_{2} \mathrm{O}_{3}$ em relação às demais, devido ao fato de que nesses ensaios houve adição de $\mathrm{Al}$ anteriormente a adição de CaSi.

A simulação termodinâmica via FactSage da composição química teve uma satisfatória aproximação com os dados obtidos pelos experimentos, validando o uso da termodinâmica computacional no estudo de inclusões não-metálicas.

O microscópio eletrônico de varredura automatizado, ASPEX-PSEM explorer se mostrou um recurso válido para identificar a distribuição dos elementos formadores das inclusões.

\section{Agradecimentos}

Os autores agradecem ao CNPq, FINEP, CAPES e UFRGS pelo apoio.

\section{REFERÊNCIAS}

1 Holappa L. Inclusion Control of Castability of Resulphurized Steels. Proceeding of 60th Ironmaking Conference; march 2001; Baltimore, USA. 2001; p.1023-1035.

2 Baptista ALB: Aspectos metalúrgicos na avaliação da usinabilidade de aços. Rem: Rev. Esc. Minas, Ouro Preto, 2002; 55(2).

\footnotetext{
* Contribuição técnica ao $69^{\circ}$ Congresso Anual da ABM - Internacional e ao 14ํㅡㄹ ENEMET - Encontro Nacional de Estudantes de Engenharia Metalúrgica, de Materiais e de Minas, 21 a 25 de julho de 2014, São Paulo, SP, Brasil.
} 


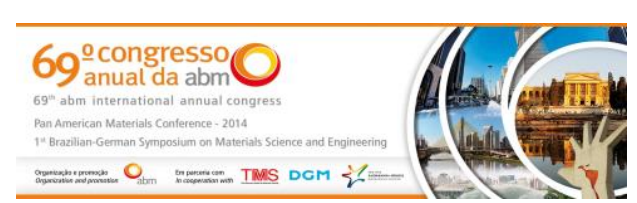

3 Larsen K, Fruehan R J. Calcium Modification of Oxide Inclusions. Transactions of the Iron \& Steel Society of AIME, 1991; 12: 125-132.

4 Bielefeldt WV. Tratamento de Inclusões Não-Metálicas com Cálcio nos Aços SAE 1141 e SAE 8620. Tese (doutorado) - Universidade Federal do Rio Grande do Sul. Escola de Engenharia. Programa de Pós-Graduação em Engenharia de Minas, Metalúrgica e de Materiais, Porto Alegre, BR-RS, 2009.

5 Kirichenco JCB. Estudo Experimental e Termodinâmico de Inclusões em Aço Ressulfurado Acalmado ao Alumínio. Dissertação (Mestrado em Engenharia de Minas, Metalúrgica e de Materiais) - Universidade Federal do Rio Grande do Sul, 2011.

6 Zhang L, Thomas BG. Inclusions in Continuous Casting of Steel. XXIV National Steelmaking Symposium, Morelia, México, p. 138-183, November, 2003.

7 Kiessling R. Non Metallic Inclusions in Steels Parts I-IV. London: The Institute of Materials, 1978.

8 Cicutti C, Madias J, Gonzalez J. Control of Microinclusions in Calcium Treated Aluminum Killed Steels. Ironmaking and Steelmaking, 1997; 24(2): 155-159.

9 Madias J. Palestra sobre Inclusões em Aços Acalmados com Alumínio e Tratados com Cálcio. Instituto Argentino de Siderurgia, 1998.

10 Beskow K, Sichen Du. Experimental Study of the Nucleation of Alumina Inclusions in Liquid Steel. Scandinavian Journal of Metallurgy, 2003; 32: 320-328.

11 Lu D. et al. Kinetics and Mechanisms of Calcium Dissolution and Modification of Oxide and Sulphide Inclusions in Steel. Ironmaking and Steelmaking, Vol. 21, n 5, 1994.

12 American Society for Testing and Materials. ASTM E 2142 - 01: Test Method for Rating and Classifying Inclusions in Steel Using the Scanning Electron Microscope. Philadelphia, PA, 2002.

13 ASM. Volume 1, Properties and Selection: Irons, Steels, and High Performance Alloys. ASM Handbook. ASM International, 1993.

14 Bielefeldt WV. Estudo do Tratamento de Inclusões com Cálcio na Fabricação do Aço SAE 8620. Dissertação (Mestrado em Engenharia de Minas, Metalúrgica e de Materiais) - Universidade Federal do Rio Grande do Sul, 134 f., 2005.

15 Dedavid BA, et al: Microscopia Eletrônica de Varredura - Aplicações e Preparação de Amostras. EDIPUCRS, 2007.

16 Gerdau. Manual de Aços Especiais. www.gerdau.com.br. Acesso em Março de 2014.

17 Holappa L, Helle AS. Inclusions Control in High Performance Steels. Journal of Materials Processing Technology, 1995; 53: 177-186.

18 Holappa L, Hamalainen M, Luikkonen M, Lind M. Thermodynamic Examination on Inclusions Modifications and Precipitation from Calcium Treatment to Solidified Steel. Ironmaking and Steelmaking, 2003; 30(2): 111-115.

19 Marcon L. Estudo de Inclusões Não-Metálicas em um Aço de Construção Mecânica. Trabalho de Diplomação. Departamento de Engenharia Metalúrgica - UFRGS, 2005.

20 Slag Atlas. 2. ed. Düsseldorf: Verlag Stahleisen GmbH, 1995.

21 Thomas BG. Tundish Nozzle Clogging - Application of Computational Models. 18rd Process Technology Division Conference, v.18. Iron and Steel Society, p. 895-912, 2001.

22 Turkdogan ET. Fundamentals of Steelmaking. The Institute of Materials, London, 1996.

23 Turkdogan ET. Metallurgical Consequences of Calcium Retention in Liquid and Solid Steel. Calcium Treatment Symposium. The Institute of Metals (now The Institute of Materials), London, p.3-13, 1988.

24 Zhang L, Thomas BG. State of the Art in Evaluation and Control of Steel Cleanliness. ISIJ International, 2003, 43(3): 271-291.

\footnotetext{
* Contribuição técnica ao $69^{\circ}$ Congresso Anual da ABM - Internacional e ao 14ํㅡㄹ ENEMET - Encontro Nacional de Estudantes de Engenharia Metalúrgica, de Materiais e de Minas, 21 a 25 de julho de 2014, São Paulo, SP, Brasil.
} 